\title{
A 1-4 GHz 4x4 MIMO Receiver with 4 Reconfigurable Orthogonal Beams for Analog Interference Rejection
}

\author{
Sajad Golabighezelahmad ${ }^{1}$, Eric Klumperink ${ }^{2}$, Bram Nauta ${ }^{3}$ \\ IC Design Group, University of Twente, Enschede, The Netherlands \\ ${ }^{1}$ s.golabighezelahmad@utwente.nl, 2e.a.m.klumperink@utwente.nl, 3b.nauta@utwente.nl
}

\begin{abstract}
A highly reconfigurable multi-beam MIMO receiver with $4 \mathrm{RF}$ inputs and 4 outputs is proposed, allowing for digital MIMO but also analog interference rejection by spatial notch filtering through 4 reconfigurable orthogonal beams. A segmented constant-Gm vector modulator with improved interference tolerance and $\mathrm{RF}$ frequency range is proposed, allowing current-domain beamforming before I-V conversion by a transimpedance amplifier. A 1-4 GHz $22 \mathrm{~nm}$ FD-SOI prototype chip achieves $>29$ spatial notch suppression for in-band interference signals. In the notches, an IIP3 of +17 $\mathrm{dBm}$ and in-band $\mathrm{B} 1 \mathrm{~dB}$ of $-12 \mathrm{dBm}$ is achieved at $44.5 \mathrm{~dB}$ gain. Sub-3dB system noise figure is achievable in the corner points of vector modulator constellation. On the circle points, noise figure degrades about $2.5 \mathrm{~dB}$. However, in-band, in-notch B1dB and IIP3 improve by $32 \mathrm{~dB}$ and $43 \mathrm{~dB}$, respectively. The chip of 0.52 $\mathrm{mm}^{2}$ active area consumes $75-115 \mathrm{~mW}$ at an LO-frequency of 1-4 $\mathrm{GHz}$ from a $0.8 \mathrm{~V}$ supply.
\end{abstract}

Keywords - Spatial filtering, Analog beamforming, Multiple-Input Multiple-Output (MIMO), Receiver, Vector Modulator, Interference rejection.

\section{INTRODUCTION}

The huge growth in wireless communication devices in scarce radio spectrum makes mutual interference increasingly a bottleneck. Spatial interference suppression by analog beamforming [1], [2], [3] can help but its Multiple-Input Single-Output (MISO) nature lacks the flexibility that Multiple-Input Multiple-Output (MIMO) systems can provide, e.g. to realize adaptive beamforming and support both spatial diversity and multiplexing gains. However, in digital MIMO, the ADCs are exposed to strong blocker signals. Adding analog spatial filtering before entering the ADCs can relax related dynamic range limits [4], [5], [6].

In this work, we present a new reconfigurable architecture supporting both digital MIMO and analog spatial filtering. Compared to prior art, performance in RF frequency range and linearity are improved in a power efficient way.

\section{Multi-beam Receiver Architecture}

Analog spatial notch filtering circuits can be added to a MIMO receiver array in different ways to reject interferers. In [4], [5], this is done by creating a beam in the direction of the interferer and subtracting a scaled version of the interferer from each of the individual antenna signals exploiting active $\mathrm{gm}$ blocks in baseband. Although frequency translated notch filtering is added to reduce RF gain somewhat, distortion in the active gm blocks poses a linearity limitation [5]. To improve interference tolerance, we propose to directly cancel the interferer current at the LNTA output in the current domain before baseband I-V conversion and gain. Conceptually, we target multi-beam MIMO for interference rejection using multiple RF phased arrays. A traditional approach for generating multi-beam is a Butler matrix as exploited in [7]. However, the beams from a Butler matrix have fixed angular positions, lacking the flexibility for arbitrary spatial interference cancellation. While a passive Butler matrix can be highly linear, it consist of bulky passive devices that are dedicated for a rather limited and fixed frequency band and not very CMOS friendly. In this paper, a flexible and wideband multi-beam receiver is implemented using multiple RF phased arrays. We propose a novel architecture targeting orthogonal beams with a programmable spatial direction, exploiting a modified constant-Gm vector modulator [3].

Conceptually, separation of the interferer and desired signals in different beams is the key goal. As illustrated in Fig.1, this can be done by a set of flexible orthogonal beams for which each output beam is aligned with the nulls of other outputs. Thus steering one of them e.g. $\mathrm{B}_{1}$ in Fig. 1 towards the interferer results in its rejection at the remaining outputs. Note that in this scenario, recovery of the individual antenna signals is still possible in the digital domain. To do so, one could simply throw away the heavily distorted output beam containing the interference $\left(B_{1}=0\right)$. By applying the inverse of the matrix representing the multi-beam $\mathrm{RF}$ front-end $\left(A_{N \times N}\right)$, the input signals are recovered and a spatial notch is formed. In this paper we will just demonstrate a programmable orthogonal beam architecture. However, once flexibly programmable analog beamforming is implemented, more complex beam patterns with multiple deep notches are possible to cancel several in-band interferers simultaneously. For this purpose, both variable phase shift and gain are needed that we achieve here using constellation points of a vector modulator. The details of vector modulator are explained in the next section.

Fig. 1 shows the block diagram of multi-beam RX. The LNTAs convert the input voltages to currents and the subsequent frequency down conversion, phase shift, and amplitude weighting all are performed in the current domain, allowing for interference cancelling by simple current summation at the input of Trans-Impedance Amplifiers (TIA). As this summing is done before I-V conversion linearity is improved. Moreover, frequency translated filtering at the output of the LNTA, which typically limits the RF bandwidth [4], [3], is not needed here. 


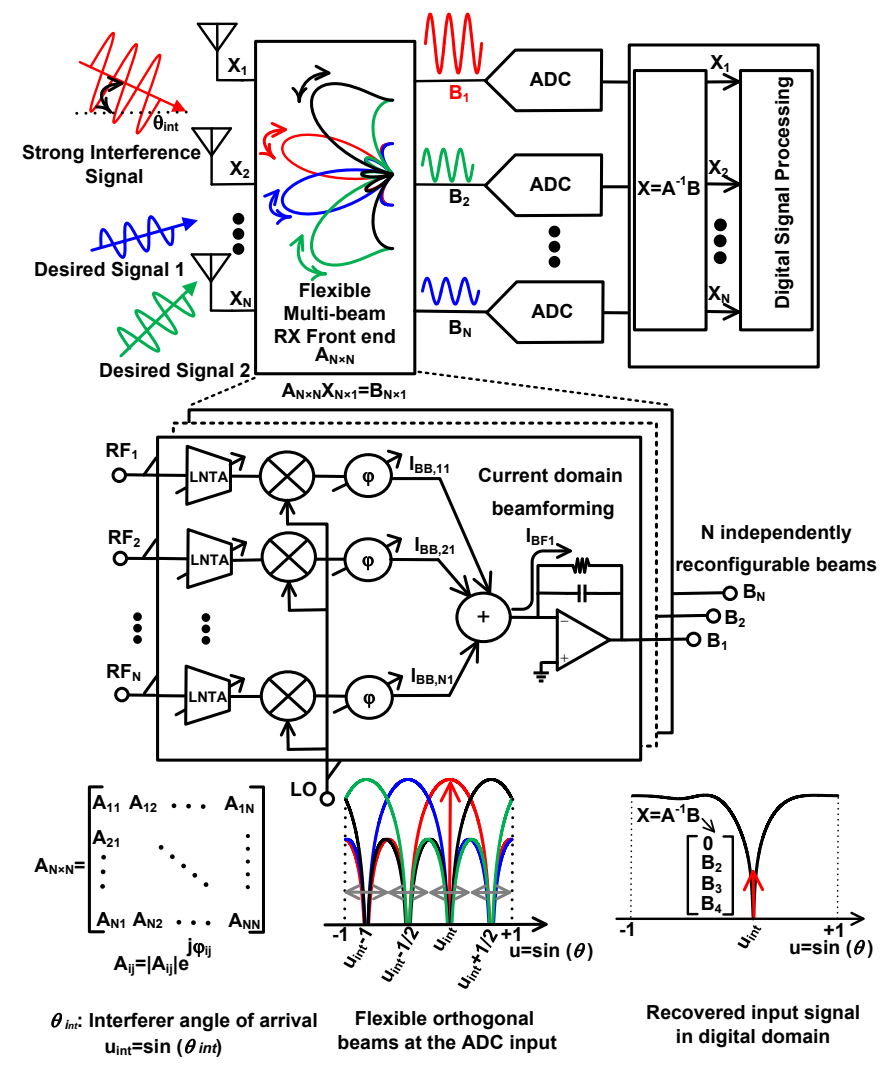

Fig. 1. Proposed multi-beam MIMO receiver using multiple RF phased arrays. Flexible orthogonal beams are created to form a notch at interference signal's angle-of-arrival.

\section{CiRCUIT IMPLEMENTATION}

A $4 \times 4$ multi-beam receiver is implemented as shown in Fig. 2, clearly showing the matrix operation. Each element of the matrix is implemented using a vector modulator, providing a complex weight for beamforming. This implementation is based on a low-power constant-gm vector modulator introduced in [3]. In this approach, a RF front-end including an LNTA followed by a 4-phase passive-mixer is sliced up into a number of binary weighted smaller cells. By static routing switches in the baseband part, each slice can contribute either to the I+, Q+, I- or Q- baseband output, producing a constellation square. A main limitation of the constant-gm vector modulator in [3] is its low gain and limited RF bandwidth. This is illustrated in Fig.3a, where the baseband signals are averaged using charge sharing at the output capacitors. This is power efficient but also results in voltage-mode operation of the passive mixer, hence the LNTA experiences a large voltage swing at its output node. High-frequency RF gain is limited by a pole at the LNTA output caused by the parasitic capacitance to ground $\left(C_{P}\right.$ in Fig. 3b) and a high output resistance.

To circumvent this bottleneck in frequency range we propose a vector modulator with current summation at the input of the TIA. Since the TIA provides low input impedance, the LNTA sees a low output impedance (as shown in Fig. $3 \mathrm{~b}$ ), leading to extended RF bandwidth of vector modulator.
Having less swing at the LNTA output also results in improved in-band output-referred linearity (OIP3). Moreover, in voltage mode operation, the amplification is limited to the intrinsic voltage gain of the LNTA [3], while a higher gain can be achieved exploiting a TIA with high feedback resistance.

Each vector modulator provides a $200 \mathrm{ohm}$ input impedance employing self-biased inverter-based LNTAs, hence, $50 \mathrm{ohm}$ low-noise input matching at each antenna interface is achieved since four vector modulators are connected to one RF input. The on-chip clock generation block produces $25 \%$ clock signals to drive the mixer switches. 15 slices are used to implement the vector modulator, which results in a $16 \times 16$ points square constellation. Using the points nearby the biggest circle fitting into the constellation square, a null depth of approximately $29 \mathrm{~dB}$ is achievable [3]. The TIA is implemented using simple inverter amplifiers using transistors with large channel lengths $(L=500 \mathrm{~nm})$ to achieve high open-loop gain and low flicker noise. Both the feedback resistor and capacitor of the TIAs are implemented in a programmable way with 3-bit digital control to provide a variable gain and baseband bandwidth for the receiver.

\section{MeAsurement Results}

A 1-4 GHz prototype chip with active area of $0.52 \mathrm{~mm}^{2}$ (see Fig. 4a) was designed and fabricated in GlobalFoundries $22 \mathrm{~nm}$ FD-SOI technology and a QFN package with test fixture on PCB was used. The measured constellation is shown in Fig.4b, where points with equal weights but variable phase nearby the biggest circle fitting into the square constellation are used to create analog beams and notches at arbitrary direction. Fig.5a shows the measured beam patterns reconfigured to produce orthogonal beams targeting a notch at $\mathrm{u}=0.25\left(\theta=14.5^{\circ}\right)$ with $\mathrm{FLO}=2.5 \mathrm{GHz}$ at $1 \mathrm{MHz}$ baseband. It is seen that a null depth of greater than $29 \mathrm{~dB}$ is achieved. To evaluate the notch suppression bandwidth, a notch is formed at broadside $(u=0)$ and RF frequency is swept at a fixed LO frequency of $2.5 \mathrm{GHz}$. As it is shown in Fig.5b, the achieved $20 \mathrm{~dB}$ notch suppression bandwidth is $2 \mathrm{GHz}$. The wideband suppression is due to immediate current-domain beamforming after voltage to current conversion by LNTA mainly because the current summation is not affected by parasitics of the circuit nodes. Fig.6a shows the total gain at $1 \mathrm{MHz}$ baseband frequency across the swept LO frequency. Equivalent single element double sideband noise figure $\left(\mathrm{NF}_{\mathrm{DSB}, \mathrm{eq}}\right)$ versus $\mathrm{LO}$ frequency is shown in Fig.6b. $\mathrm{NF}_{\mathrm{DSB}, \mathrm{eq}}$ is measured using single excitement method introduced by [4], [5]. Noise figure is measured both on the biggest circle fitting into the square constellation and on its corner points where all the vector modulator cells are in-phase and contribute to the signal gain, hence improving the NF by $2.5 \mathrm{~dB}$. The best achievable system noise figure hence occurs in the corner points (providing analog beams and notches at $\mathrm{u}=0, \mathrm{u}=1 / 2$, $\mathrm{u}=-1 / 2, \mathrm{u}=1$ and digital beamforming can be performed later at arbitrary direction in the digital domain). Taking into account the SNR improvement of $6 \mathrm{~dB}$, a sub-3dB system noise figure can be obtained. When exploiting analog beamforming 


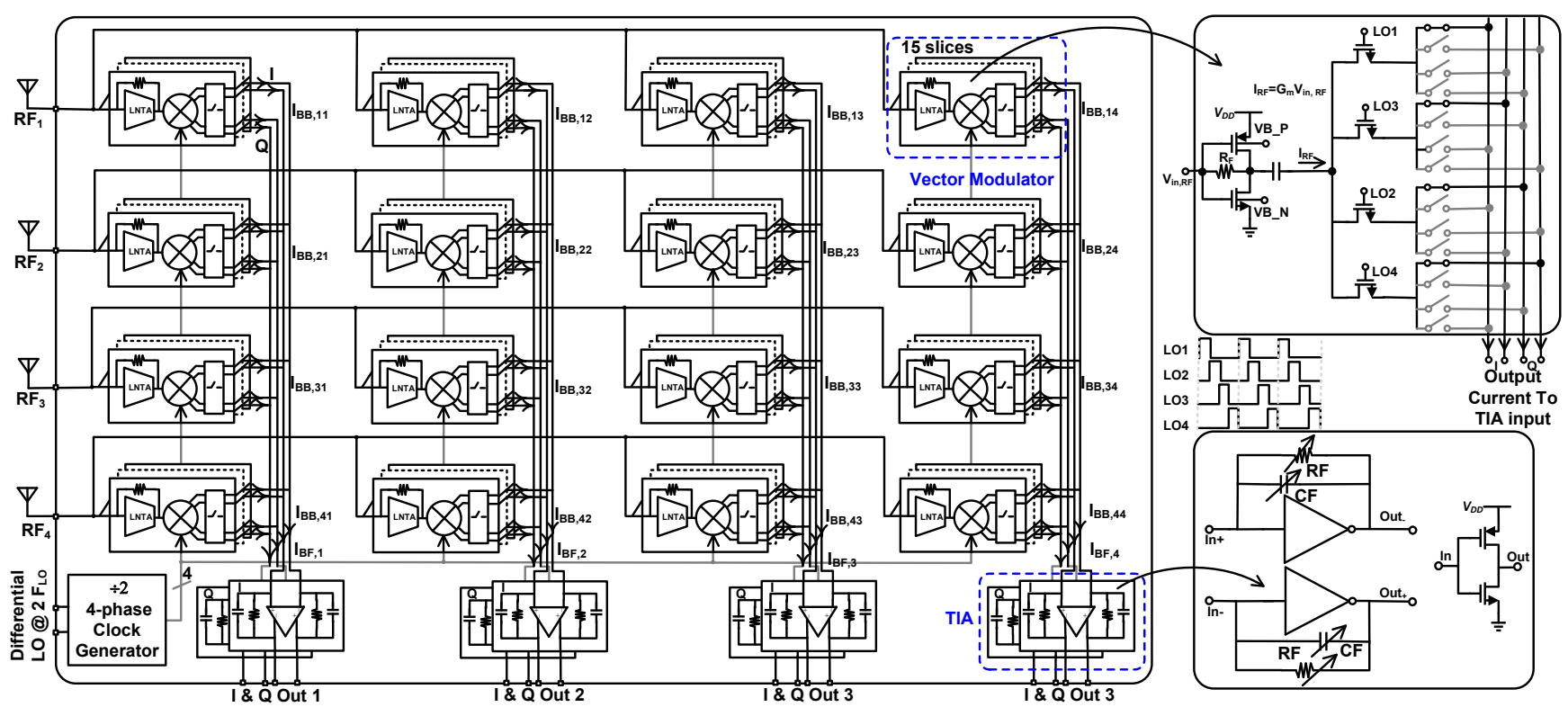

Fig. 2. Proposed multi-beam $4 \times 4$ MIMO receiver employing constant-gm vector modulators with current summing (interference rejection) at the input of the TIA.

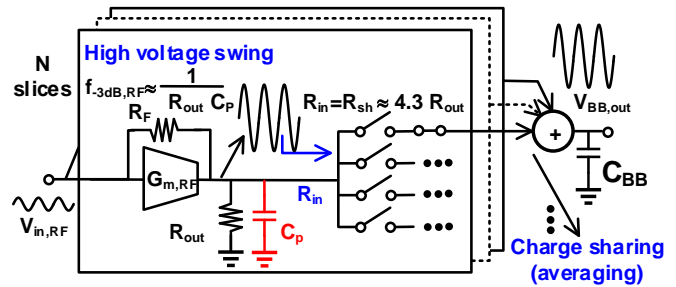

(a)

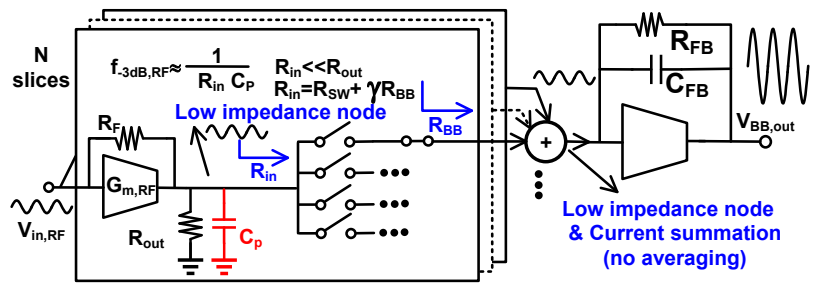

(b)

Fig. 3. (a) Constant-gm vector modulator with beamforming by charge sharing, i.e. I-V conversion with averaging [3]; (b) Proposed constant-gm vector modulator with current summing. This results in more achievable gain, better linearity, and RF frequency range.

with points nearby the circle in Fig. $4 \mathrm{~b}$ to realize notches in arbitrary directions, the noise figure degrades about $2.5 \mathrm{~dB}$ as mentioned above, but linearity improves much more, i.e. Spurious Free Dynamic Range benefits.

In-beam/in-notch IIP3 versus offset frequency of first tone are presented in Fig.7a. The results show $+3 \mathrm{dBm}$ out-of-band linearity in the main beam direction, limited by the linearity of the LNTA. In-band IIP3 of $-26 \mathrm{dBm}$ is achieved, corresponding to OIP3 of $+18.5 \mathrm{dBm}$ (44.5 dB gain). In measuring in-notch IIP3, it is assumed that a two-tone interfere is arriving at the receiver array from the spatial notch direction. Since the intermodulation product is suppressed by the notch filtering, the in-band IIP3 is improved to +17 $\mathrm{dBm}$ for in-notch incidence. This IIP3 remains approximately constant across the offset frequency, which fits to the wide
$20 \mathrm{~dB}$ rejection bandwidth observed in Fig.5b. Fig.7b shows the conversion gain for the desired signal in terms of in-band, in-beam/in-notch blocker power level; putting a notch at the blocker's angle improves the blocker tolerance about $32 \mathrm{~dB}$, achieving a high $\mathrm{B} 1 \mathrm{~dB}$ of $-12 \mathrm{dBm}$.

Table1 summarizes the performance of the chip and benchmarks it with the state-of-the-art. The wideband RF bandwidth of the receiver covers the frequency range from 1 $\mathrm{GHz}$ to $4 \mathrm{GHz}$. The in-band, in-beam OIP3 as well as in-band, in-notch IIP3 values are the highest among MIMO receivers. Moreover, in-notch $\mathrm{B} 1 \mathrm{~dB}$ of $-12 \mathrm{dBm}$ at $44.5 \mathrm{~dB}$ gain is the best achieved blocker tolerance for MIMO receivers. Taking into account $6 \mathrm{~dB}$ SNR improvement due to the analog beamforming, the receiver achieves sub- $3 \mathrm{~dB}$ noise figure at the corner points. The noise performance is slightly worse than digital MIMO chips presented in [4], [5], but this is compensated by very significant improvements in linearity. The chip consumes $75-115 \mathrm{~mW}$ at LO frequency of 1-4 $\mathrm{GHz}$, operating at faster clock speed compared to prior art, demonstrating power-efficient implementation considering the direct dependency of dynamic power consumption on LO clock frequency.

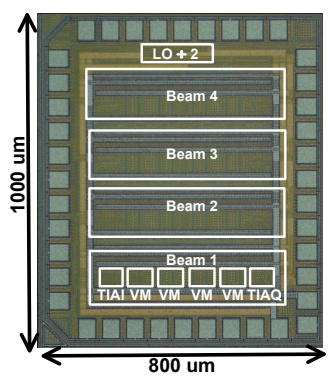

(a)

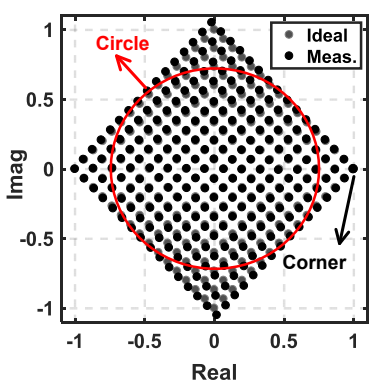

(b)
Fig. 4. (a) Die micrograph; (b) Constellation points. 
Table 1. Performance summary and comparison with state-of-the-art.

\begin{tabular}{|c|c|c|c|c|c|c|c|}
\hline Architecture & $\begin{array}{c}\text { ISSCC11 } \\
{[1]}\end{array}$ & $\begin{array}{c}\text { ISSCC13 } \\
{[2]}\end{array}$ & $\begin{array}{c}\text { JSSCC17 } \\
{[3]}\end{array}$ & $\begin{array}{c}\text { RFIC16 } \\
{[6]}\end{array}$ & $\begin{array}{c}\text { ISSCC16 } \\
{[4]}\end{array}$ & $\begin{array}{c}\text { ISSCC17 } \\
{[5]}\end{array}$ & This work \\
\hline Process & $65 \mathrm{~nm}$ & $65 \mathrm{~nm}$ & $65 \mathrm{~nm}$ & $65 \mathrm{~nm}$ & $65 \mathrm{~nm}$ & $65 \mathrm{~nm}$ & $22 \mathrm{~nm}$ FD-SOI \\
\hline Active area $\left(\mathrm{mm}^{2}\right)$ & 0.44 & 0.97 & 0.2 & 3.8 & 1.69 & 1.44 & 0.52 \\
\hline Supply (V) & 1.2 & 1.2 & 1 & $1.3-1.5$ & 1.2 & 1.2 & 0.8 \\
\hline Array elements & $4 \times 1 \mathrm{MISO}$ & $4 \times 1$ MISO & $4 \times 1$ MISO & $4 \times 4$ MIMO & $4 \times 4$ MIMO & $4 \times 4$ MIMO & $4 \times 4$ MIMO \\
\hline RF Frequency $(\mathrm{GHz})$ & $1-4$ & $0.6-3.6$ & $1-2.5$ & 10 & $0.1-1.7$ & $0.1-3.1$ & $1-4$ \\
\hline Total Gain $(\mathrm{dB})$ & 16 & -1 & 12 & 14 & 41 & 43 & 44.5 \\
\hline Single Element $\mathrm{NF}_{\mathrm{DSB}, \mathrm{eq}}(\mathrm{dB})$ & 10 & $5-8$ & 6 & 9.5 & $2.2-4.6$ & $3.4-5.8$ & $\begin{array}{l}\text { 10.9-11.6(Circle) } \\
\text { 8.4-9.5(Corner) }\end{array}$ \\
\hline Max.Spatial Suppression(dB) & $>20$ & - & 29 & 32 & 32 & $51-56$ & $>29$ \\
\hline 20 dB Spat. Supp. Band. (MHz) & - & - & - & $100(1 \%)$ & $15(3 \%)$ & $\begin{array}{c}320 @ 500 \\
\mathrm{MHz} \\
(64 \%)\end{array}$ & $\begin{array}{c}2000 @ 2.5 \\
\mathrm{GHz} \\
(80 \%)\end{array}$ \\
\hline In-band/in-beam OIP3(dBm) & +15 & - & 13 & - & 0 & +11 & +18.5 \\
\hline In-band/in-notch IIP3(dBm) & - & +9 & +20 & - & -7 & +1 & +17 \\
\hline Out-of-band IIP3 (dBm) & - & +20 & +5 & - & +11 & -5 & +3 \\
\hline In-notch B1dB (dBm) & - & - & - & - & - & -25 & -12 \\
\hline Power (4 Elements, mW) & $308 @ 2.5 \mathrm{GHz}$ & $\begin{array}{c}66-195 \\
40 \mathrm{~mW}+43 \\
\mathrm{~mW} / \mathrm{GHz}\end{array}$ & $\begin{array}{c}26-36 \\
19 \mathrm{~mW}+6.7 \\
\mathrm{~mW} / \mathrm{GHz}\end{array}$ & $\begin{array}{c}145 \\
\text { (1 Elem.) }\end{array}$ & $148 @ 0.5 \mathrm{GHz}$ & $\begin{array}{c}116-147 \\
115 \mathrm{~mW}+10 \\
\mathrm{~mW} / \mathrm{GHz}\end{array}$ & $\begin{array}{c}75-115 \\
62 \mathrm{~mW}+13.2 \\
\mathrm{~mW} / \mathrm{GHz}\end{array}$ \\
\hline
\end{tabular}

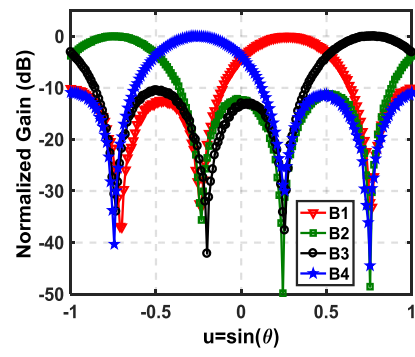

(a)

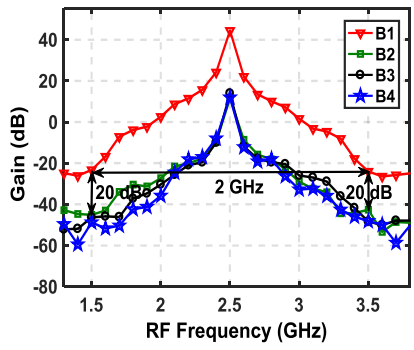

(b)
Fig. 5. (a) Measured beam patterns @ $\mathrm{FLO}=2.5 \mathrm{GHz}$ with a notch at $\mathrm{u}=0.25$; (b) $20 \mathrm{~dB}$ notch suppression bandwidth at broadside notch $(u=0)$.

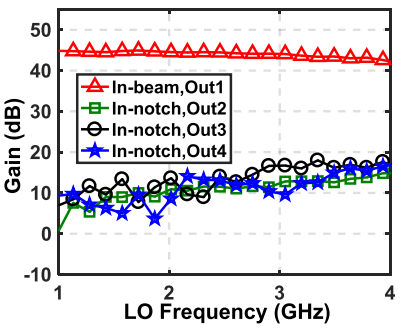

(a)

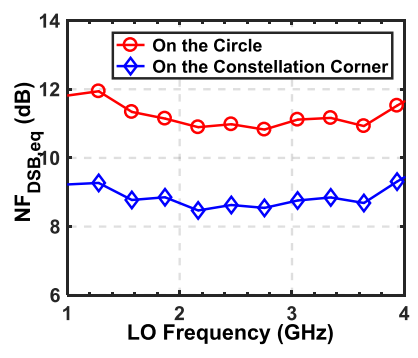

(b)
Fig. 6. (a) Total conversion Gain and (b) $\mathrm{NF}_{\mathrm{DSB}, \mathrm{eq}}$ across LO frequency.

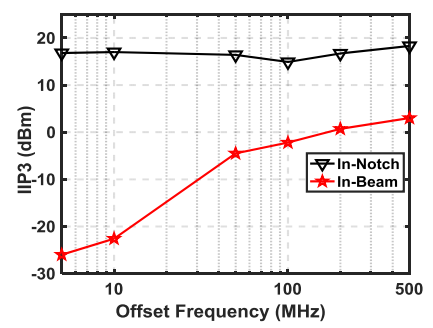

(a)

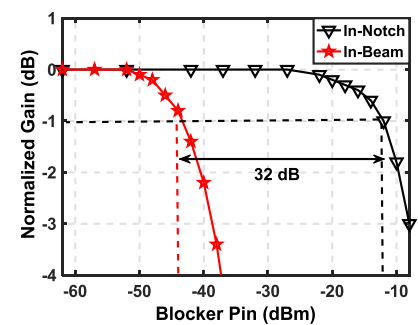

(b)
Fig. 7. (a) In-beam/in-notch IIP3 as function of offset frequency of first tone; (b) Conversion gain for desired signal versus in-band, in-beam/in-notch blocker input power.

\section{CONCLUSION}

In this paper, a highly flexible and reconfigurable multi-beam receiver was presented for spatial in-band blocker rejection. The maximum RF operating frequency is extended up to $4 \mathrm{GHz}$. Interference suppression early in the receiver chain before voltage amplification improves its linearity performance; in-band, in-notch IIP3 and B1dB of $+17 \mathrm{dBm}$ and $-12 \mathrm{dBm}$, respectively, at $44.5 \mathrm{~dB}$ gain.

\section{ACKNOWLEDGMENT}

The authors would like to thank Gerard Wienk for CAD assistance and Henk de Vries for measurement setup. The authors also thank GlobalFoundries for supporting chip fabrication.

\section{REFERENCES}

[1] M. C. M. Soer et al., "A 1.0-to-4.0GHz $65 \mathrm{~nm}$ CMOS four-element beamforming receiver using a switched-capacitor vector modulator with approximate sine weighting via charge redistribution," in ISSCC, Feb 2011, pp. 64-66.

[2] A. Ghaffari et al., "Simultaneous spatial and frequency-domain filtering at the antenna inputs achieving up to $+10 \mathrm{dBm}$ out-of-band/beam P1dB," in ISSCC, Feb 2013, pp. 84-85.

[3] M. C. M. Soer et al., "Beamformer With Constant-Gm Vector Modulators and Its Spatial Intermodulation Distortion," IEEE J. Solid-State Circuits, vol. 52, no. 3, pp. 735-746, March 2017.

[4] L. Zhang et al., "A scalable $0.1-$ to- $1.7 \mathrm{GHz}$ spatio-spectral-filtering 4-element MIMO receiver array with spatial notch suppression enabling digital beamforming," in ISSCC, Jan 2016, pp. 166-167.

[5] — "A 0.1-to-3.1GHz 4-element MIMO receiver array supporting analog/RF arbitrary spatial filtering," in ISSCC, Feb 2017, pp. 410-411.

[6] S. Jain et al., "A 10GHz CMOS RX frontend with spatial cancellation of co-channel interferers for MIMO/digital beamforming arrays," in IEEE Radio Frequency Integrated Circuits Symp., May 2016, pp. 99-102.

[7] A. Tork et al., "Reconfigurable X-Band 44 Butler array in 32nm CMOS SOI for angle-reject arrays," in IEEE MTT-S International Microwave Symp., May 2016, pp. 1-4. 